\title{
Expansion of the supergranular magnetic network through the solar atmosphere
}

\author{
T. Aiouaz ${ }^{1}$ and M.P. Rast ${ }^{1,2}$ \\ ${ }^{1}$ High Altitude Observatory, National Center for Amospheric Research, Boulder, CO, USA \\ email: aiouaz@ucar.edu \\ ${ }^{2}$ Laboratory for Atmospheric and Space Physics, University of Colorado, Boulder, CO, USA
}

\begin{abstract}
The solar magnetic field that extends through the chromosphere is thought to expand through the transition region into the corona. The strong flux concentrations are located within the boundaries of supergranular convection cells. These boundaries form network lanes, observed in emission lines as bright lanes with varying width throughout the solar atmosphere. These network field concentrations are surrounded by mixed-polarity magnetic field with a scale of the granule diameter, as suggested by observations. We use potential magnetic field extrapolations on synthetic magnetograms to study the magnetic network topology and the effects of background magnetic field on the network expansion through the solar atmosphere. We find that the background magnetic field has a considerable effect on the ratio of network area over field of view. Furthermore we find that the expansion of the network boundaries with height deviate significantly from well-assumed funnel model expansion.
\end{abstract}

Keywords. Sun: magnetic fields, Sun: atmosphere, Sun: chromosphere

\section{Introduction}

The magnetic field in the solar atmosphere depends on the photospheric flux consisting of magnetic elements. The plasma flow carry magnetic field lines to the edges of the supergranular convection cells. The supergranular convection cell boundaries are characterized by a larger magnetic flux than the cell interiors. The upward extension of these boundaries are observed as network lanes, which is seen in Extreme Ultra-Violet emission lines as bright lanes in intensity (Reeves 1976). Moreover it is believed that the network is the basic channel of the energy responsible for heating the corona and accelerating the solar wind (e.g. Reeves 1976; Gabriel 1976; Marsch \& Tu 1997; Aiouaz et al. 2005).

Therefore, a precise knowledge of the expansion of network lanes throughout the solar atmosphere can provide constraints on the relative weights to be attributed to the different terms in the steady state energy budget in the solar atmosphere. Up to now only theoretical modeling of height variation of flux tube cross section (e.g. Gabriel 1976) has been used to predict the network shape. Some others study using observations were realized to measure the variation of the network width with height (e.g. Patsourakos et al. 1999), but difficulties are usually encountered due to limited resolution, and due to ambiguous intensity threshold methods used to define the network. It is therefore necessary to elaborate a method that calculate the variation of the network lanes through the atmosphere. This paper is intended to give a coverage of the effect of boundary conditions, and internetwork magnetic field on three-dimensional network magnetic topology.

\section{Synthetic magnetograms}

Supergranular cells are distributed fairly uniformly over the solar disk and occurs on the scale of approximately 20-30 Mm horizontally. In this model supergranular cells have 

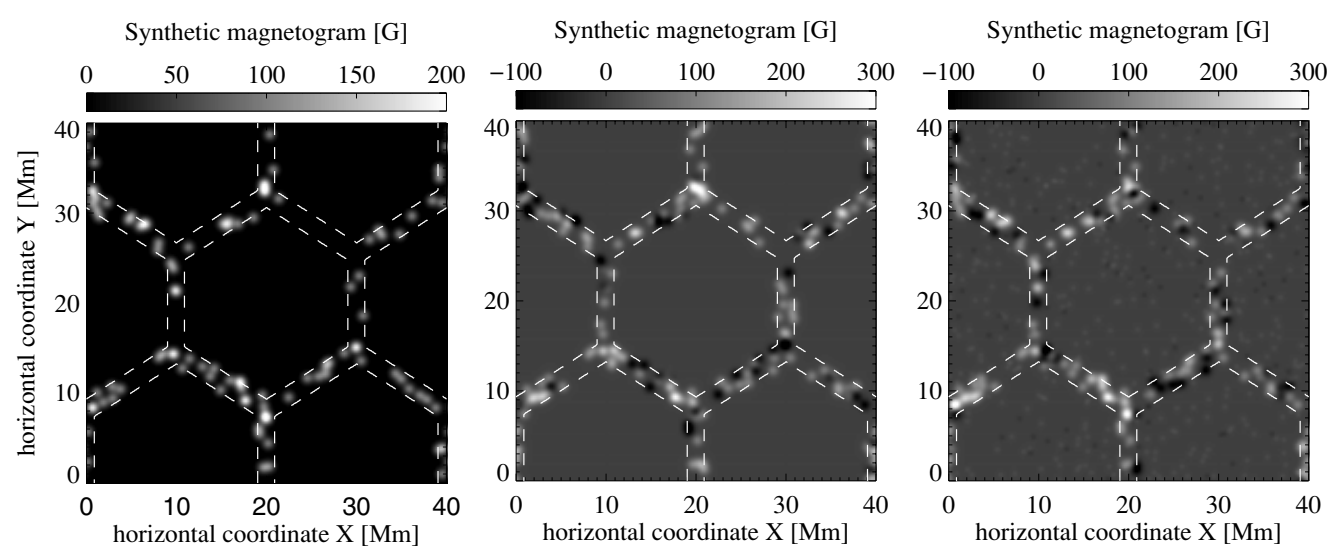

Figure 1. Synthetic magnetograms (see section 2).

hexagonal shape. The size of the synthetic magnetogram is $40 \mathrm{Mm} \times 40 \mathrm{Mm}$. The network lanes (see Figure 1) around and between the supergranular cells are $2 \mathrm{Mm}$ wide. The so defined network lanes are the seed areas for a random distribution of strong network magnetic field concentrations.

We produced three different topology for the magnetic field with increasing magnetic complexity as displayed in Figure 1. The first case shows unipolar magnetic field concentrations randomly distributed in the network lanes (left panel in Figure 1), the second case shows concentrations of mixed polarities randomly placed in the network lanes (middle panel in Figure 1). In the two previous cases, no magnetic field concentration is seeded within the supergranular cells. The last case (right panel in Figure 1) is similar to the second case for the magnetic topology within the network lanes (mixed polarities), but a background field has been added. This background field consist of weak magnetic field concentrations of mixed polarities randomly distributed within the cells. Special care is made to keep the total flux of the background field equal to zero.

The magnetic field concentrations are spatially defined as two dimensional Gaussian distributions. The FWHM of the distributions is $0.5 \mathrm{Mm}$ and $1 \mathrm{Mm}$ for the weak and strong magnetic field concentrations respectively. The ratio of amplitudes of strong over weak concentrations is 100 . The number of randomly distributed strong magnetic concentrations within the network is 100 positives for the unipolar case, and 150 positives and 50 negatives for the two other cases. The number of randomly distributed weak magnetic concentrations for the background field in the last case is 250 positives and 250 negatives.

\section{The network expansion model}

At present, the solar vector magnetic field is routinely measured from the Zeeman effect in the photosphere, so we have to rely on field extrapolation methods to infer properties of the coronal field. Simple fields, such as potential magnetic fields, are broadly applicable and still widely invoked in the literature. Even though it is an extreme simplification which does not take in account the MHD effects and radiative transfer near the solar photosphere, its simplicity allow a complete study of magnetic topology over a wide range of resolution and boundary conditions, which is nowadays out of reach with the current computing facilities for the more complex extrapolation methods. 

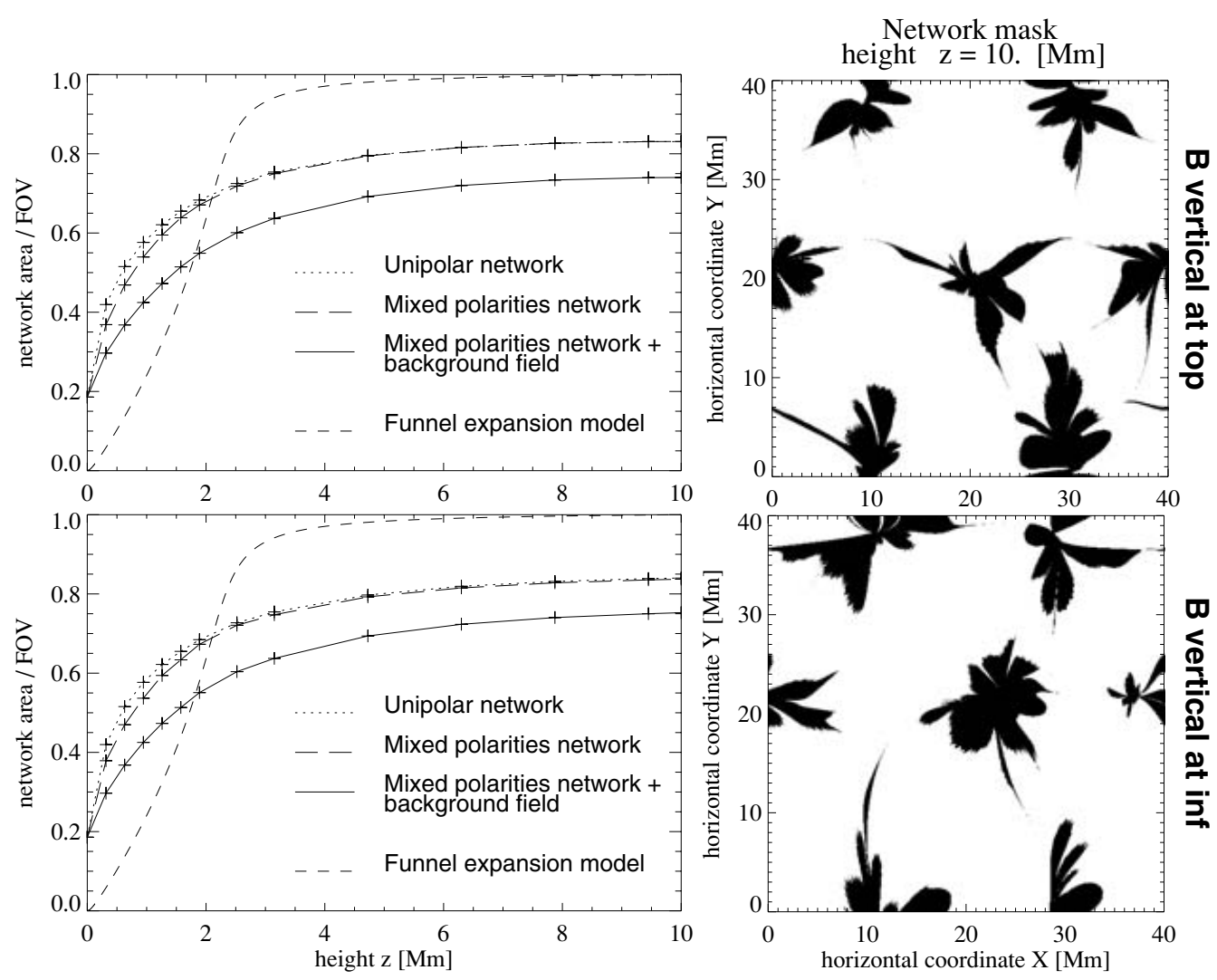

Figure 2. Results of the network expansion model (see section3-4).

To determine the magneto-static potential field, we solve the linear equation, $\nabla \times \mathbf{B}=$ 0, using Fourier transforms where the component perpendicular to photospheric plane has an exponential increasing and decreasing term, as first suggested by Alissandrakis (1981). The inference of a potential magnetic field in the corona is only constrained by the values at the photospheric boundary, and thus, mathematically, represents a boundary problem. Additionally to periodic boundary conditions in the horizontal directions, boundary conditions in the vertical direction need to be set to fully determined the solution of the boundary value problem. For the lower boundary, at the plane $z=0$, we assumed the vertical component of the magnetic field to be given by the synthetic magnetograms. For the upper boundary, we will assume the magnetic field to be vertical after a certain height, which is a reasonable assumption regarding numerous models of the magnetic field in the solar corona. We calculate and discuss two different cases, in the first the magnetic field is vertical at the top of the computational domain, in the second the magnetic field is "vertical at infinity", this is the case assumed by Alissandrakis (1981).

Once all three components of the magnetic field are computed, we are able to follow the expansion of the network. At any chosen height for each pixel of the map we trace back the corresponding magnetic field line to the photospheric plane. We define as network (white pixels in left panels in Figure 2) each pixel with a corresponding magnetic field line routing back to the seed area defined previously (see Figure 1) as network lanes. We obtain thus network mask at any chosen height, and can thus follow the expansion 
of the so defined network lanes throughout the atmosphere as exposed in Figure 2. The left panels in Figure 2 show the variation of percentage of network (white areas) over the total field of view (FOV) throughout the atmosphere. On each of these left panels is represented the case of the unipolar network (dotted line), mixed polarities within the network (long dashed line), and the case of mixed polarities within the network with a background field (solid line). Additionally the funnel expansion model is plotted with dashed line for qualitative comparisons. The right panels shows, as example, the form of the network at the top end of the computational domain $(z=10 \mathrm{Mm})$ in the case of the network with mixed polarities magnetic field concentrations. The upper panels show the case where the magnetic field is vertical of the top boundary, and the lower panels the case where the magnetic field is "vertical at infinity".

\section{Results and conclusions}

The left panels show that after a height of approximately $z=3 \mathrm{Mm}$ the network expansion for the unipolar case (dotted line) and the mixed polarities case (long dashed line) join together to stay almost identical until the top boundary. The mixed polarities within the network in the second case imply the presence of loops within the network. This results shows clearly that the effect of network loops on the network expansion, should no longer be expected at heights greater than $z=3 \mathrm{Mm}$. This result is of major importance because it give a new constraint for observations aimed to study the magnetic network topology.

Furthermore the left panels show that the case with a background field is different from the two previous case from the photospheric level until the end of the domain. It shows clearly that the presence of a background field affect significantly the expansion of the network. It shows less network than the two previous cases throughout the atmosphere with $10 \%$ less network at the top end. One has to remind that special care was taken to keep the total flux of the background field equal to zero. This lack of so defined network shows the effect of the weak field concentration seeded (randomly) next to the network lanes. These weak concentrations prevent for some of the network field to reach the upper boundary of the domain. Thus small loops appear with one feet within the network and the other within the cell area.

Finally the three previous considered curves show clearly differences in shape with the point charge funnel expansion model. This funnel model, derived from the well known Gabriel (1976) model, has been considered mistakenly until now has the reference model for the expansion of the network.

The differences in network shape between the right upper panel and the right lower panel, shows the significant effect of the top boundary condition on the topology of magnetic field. Nevertheless the upper left panel and the lower left panel are almost identical, showing how strong and stable are the results on the expansion of the network through this model.

\section{References}

Aiouaz, T., Peter, H., \& Keppens, R., 2005, A\&A442, L35

Alissandrakis, C. E., 1981, A\&A100, 197

Gabriel, A. H., 1976, Phil. Trans. R. Soc. Lond. A. 281(1304), 339

Marsch, E., Tu C.-Y., 1997, Sol. Phys. 176, 87

Patsourakos, S., Vial, J.-C., Gabriel, A. H., \& Bellamine, N., 1999, ApJ 522, 540

Reeves, E., 1976, Sol. Phys. 46, 53 